\title{
A Refutation of Goodman's Type-Token Theory of Notation
}

\author{
John DILWORTH ${ }^{\dagger}$
}

\begin{abstract}
In Languages of Art, Nelson Goodman presents a general theory of symbolic notation. However, I show that his theory could not adequately explain possible cases of natural language notational uses, and argue that this outcome undermines, not only Goodman's own theory, but any broadly type versus token based account of notational structure.

Given this failure, an alternative representational theory is proposed, in which different visual or perceptual aspects of a given physical inscription each represent a different letter, word, or other notational item. Such a view is strongly supported by the completely conventional relation between inscriptions and notation, as shown by encryption techniques etc.
\end{abstract}

In Chapter 4 of his book Languages of Art, ${ }^{1}$ Nelson Goodman presents a general theory of symbolic notation, two of whose main syntactic requirements, of disjointness and finite differentiation, are "...met by our alphabetical, numerical, binary, telegraphic, and basic musical notations; and by a wide variety of other describable notations..." $(1968,140)$.

However, I shall show that his theory could not adequately explain some possible cases of natural language notational uses, and argue that this outcome undermines, not only Goodman's own theory, but any broadly type versus token based account of notational structure.

Following C. S. Peirce, ${ }^{2}$ it has become standard to distinguish a word or character type, such as the letter 'a', from any particular inscription or token of that type, such as a particular 'a'-shaped, printed mound of ink on a page. For his own nominalist reasons, Goodman prefers to translate the usual type-token terminology into a nominalist substitute, in two stages: first the letter 'a' itself - the type - is regarded as a class of particular inscriptions or marks, and then he treats those "...so-called tokens of a type as replicas of one another." (1968, 131, fn.3) However, nothing in what follows will depend on whether the type-token distinction is construed in standard or nominalistic ways. Also the points to be made arguably would equally apply to a kind-instance or universal-particular interpretation of the distinction. ${ }^{3}$

$\dagger$ Department of Philosophy, Western Michigan University, Kalamazoo, MI 49006, USA.

${ }^{1}$ Goodman 1968. Goodman draws on his earlier account in The Structure of Appearance, 1966.

${ }^{2}$ See Collected Papers of Charles Sanders Peirce, ed. Hartshorne and Weiss 1931-58, vol. 4, para. 537; vol. 8, para. 334; vol. 2, para. 243-6.

${ }^{3}$ Or at least, insofar as a broadly extensionalist account may be given of those contrasts, as presumably would generally be agreed to for merely notational or syntactic items.

Dialectica Vol. 57, N 3 (2003), pp. 330-336 
Goodman's syntactic disjointness condition is that

"...no mark [token] may belong to more than one character [type]", and he adds "That the characters must thus be disjoint may not seem very important or striking; but it is an absolutely essential and, I think, rather remarkable feature of notations." (1968, $133){ }^{4}$

It is this syntactic condition on which I shall concentrate my remarks. ${ }^{5}$

What I shall show is that there could be plausible cases in which a single physical inscription or token could be an inscription of two or more distinct letters or words. ${ }^{6}$ For example, an enterprising night-club owner who also sells stationery supplies might commission a sign, on which the word 'din' - advising potential customers of the high level of noise inside - is painted on a clear plastic backing. But the owner also knows that if that same sign is exhibited so that it can also be seen from the other, reverse side, that same painted lower-case inscription will also serve as an inscription of the distinct word 'nib' - an example of the owner's stationery supplies.

Such a double sign creates an insoluble problem for the type view, for the following reason. According to the type view, distinct words or letters must be associated with disjoint sets of their respective tokens, for if the sets had a non-null intersection, with one or more tokens belonging to both types, then that would merely prove that the two types - and hence the two words themselves on this view - were not distinct after all. Thus disjointness of extension for two types of the same general kind - such as both being words - is a necessary condition of their genuinely being distinct types. ${ }^{7}$ However, in the case of any simultaneously doubly used inscription, it would, on a type view, count as a token of both types after all, and hence undermine the distinctness of the two words and of their corresponding types. ${ }^{8}$

Now with this particular example a defender of the received type view might seek to proceed as does Goodman in discussing the foundations of notational systems. ${ }^{9}$ Of a related example involving letters Goodman says:

But what of a mark that, equivocally, reads as different letters when placed in different contexts at different times? Disjointness is violated if any mark belongs to two different characters, whether at the same time or at different times..$^{10}$ Thus if the alphabet is

${ }^{4}$ This syntactic disjointness condition must be distinguished from any putative semantic disjointness condition, for as Goodman points out $(1968,152)$, such a semantic requirement would rule out most natural languages as notational systems.

${ }^{5}$ Goodman's other main syntactic condition of finite differentiation is, roughly speaking, that tokens of each type must clearly be distinguishable from tokens of some other type: see Goodman 1968, 135-6. But his account has some technical flaws, as pointed out and corrected by Kendall Walton, 1971.

${ }^{6}$ Goodman, ibid. 137-39, gives several examples of doubly functioning letters. The following double-word example is my own, introduced so as to permit discussion of some issues additional to those arising from Goodman's own examples.

${ }^{7}$ Similarly, Goodman stresses that "...no mark [inscription] may belong to more than one character", and, as previously noted, "That the characters must thus be disjoint... is an absolutely essential and, I think, rather remarkable feature of notations." (Goodman, ibid. 133).

${ }^{8}$ Actually a similar criticism would apply, though in a less apt form, to virtually any word-inscription perceivable from both its obverse and reverse sides (except for those such as the word 'mom' that look the same from both sides). In most cases the reverse side would not be perceivable as a distinct word, in which case the incompatibility would be that of an inscription that both is (on one side) and is not (on the other side) a token of a given word.

${ }^{9}$ Goodman, ibid.

10 Here Goodman invokes the same type-theoretic requirement of disjointness for letters and other characters as I did above. 
to qualify as a notation, not such enduring marks but, rather, unequivocal time-slices of them must be taken as members of the characters - that is, as inscriptions of letters. ${ }^{11}$

A creative defender of the received view might try to make a corresponding point about my 'din/nib' example, except that in this case she would be forced to distinguish two distinct sides of the three-dimensional painted marks, or 'space-slices' of them. ${ }^{12}$ Thus her view would be that in this case it is the distinct space-slices of the painted sign that comprise distinct tokens of the words 'din' and 'nib', and in that way avoid violation of the disjointness requirement.

Now such a view would be acceptable but unnecessary in the case of some double signs, such as a billboard that has one textual sequence on a paper backing affixed to one of its sides, and a completely different textual sequence and backing - both physically distinct marks and backings, and distinct letters and words - affixed to the other side. In such a case the relevant physical inscriptions or marks are indeed physically distinct, and so there would be no need to invoke the exotic idea of 'space-slices'.

On the other hand, in the case of my 'din/nib' example the idea is much more problematic. For after all, I did not specify how thick the painted marks were that were applied to the clear plastic: if they were only one molecule thick, then it is not obvious that any clear sense can be given to the idea of a 'space-slice' of a molecule and its neighbors, which presumably would at least require both that each molecule has a 'front' and a 'back' side, and also that each be spatially divisible, at least in principle if not in practice - for in the case of the more established notion of a time-slice, at least we understand both in principle and practice what it would be to temporally divide a mark in that way.

Thus I conclude that the 'space-slice' defense does not work because of the incoherence, or at least fundamental unclarity, of the concept as applied to at least some potentially physically real inscriptions. In such a potential case, there is no gainsaying that it would be the same physical marks that one would see from either side of the sign, and therefore that the inscriptions one thus saw could not be tokens of distinct word-types. ${ }^{13}$

In any case, it is arguable that time-slice or space-slice defenses, even in cases where both concepts are conceptually or operationally clear, are no better than a rhetorical maneuver of changing the subject when under attack. For the original issue was of how a normal inscription, that was a temporal and spatial continuant, could be an inscription of two distinct linguistic types. That problem has not been solved, but merely postponed or shelved, by instead moving to talk of ontologically distinct entities such as temporal or spatial slices.

Goodman himself does briefly suggest another way of trying to deal with an example such as mine, as follows:

11 Ibid., 139.

12 Time-slices would be ruled out because of the possibility of simultaneous viewing of both sides of the sign, either by different people, or by one person using mirrors etc. to simultaneously see both the obverse and reverse sides.

13 A related example involving non-simultaneously seeable words would be the word inscription 'mop', which when spatially inverted becomes the word inscription 'dow'. But each such spatial presentation still involves numerically the same physical inscription, and hence it provides another counter-example to Goodman's disjointness condition. (Also, even in this example, simultaneous seeing of both words would be possible through use of an inverting series of mirrors and lenses, hence undermining attempted 'space-slice' or 'time-slice' interpretations of the case). 
Where a mark is simultaneously multiply oriented - legitimately subject to different readings from different directions at once - it may belong to a character consisting of all marks having the same multiple orientation and readings. ${ }^{14}$

Presumably then Goodman's view of my 'din/nib' case would be that it is an inscription of a single word, which consists of all marks having the same multiple orientation and readings ${ }^{15}$ - such as all roughly similar, equivalently painted and multiply oriented signs.

But Goodman is, on one natural interpretation of his remark, trying to have his cake and eat it too here. If his escape route is to claim that the inscription is in fact not a token of the two words 'din' and 'nib' at all, but instead that it is a token of a different, single word, whose token is multiply oriented, then only a single reading of that token as that word is possible: that token must be read as that same new word, no matter which side of the inscription is viewed by a reader of it, so that the original two different readings of the inscription can play no part in this new syntactic construal of the inscription. Thus any suggestion (whether intended or implied) that the new word could somehow take over or inherit the original two readings of the inscription must be rejected.

On the other hand, in his phrase "having the same multiple orientation and readings" Goodman may merely be identifying which inscriptions would qualify as tokens of his new word - namely those that (necessarily incorrectly) might be thought to be simultaneously tokens of the two distinct words 'din' and 'nib'. In this case his phrase is unobjectionable, but the starkly ad hoc nature of his maneuver is much more apparent.

Specifically, there are the following problems with this approach. First, we have no idea which word this new word is, beyond the bare fact of having been given directions as to how to recognize members of its associated token set or extension. (Also of course, we have no clue as to the semantics of this supposed word, given its necessary non-connection with the words 'din' and 'nib'). Thus Goodman could offer no explanation of obvious facts such as that the night-club owner could in fact both conceive of and actually have built such a dual-purpose sign, plus the fact that his customers could indeed read and understand the sign to be the normal word 'din' on one of its sides, and the distinct normal word 'nib' on the other side.

But there is a more damaging criticism of Goodman's potential proposal, namely that this supposed new word is not only missing from standard English, but that it does not even qualify as a possible new word in English, since one of its supposed letters is not itself an English letter - namely the letter that looks like a ' $b$ ' from one side of the sign, but like a 'd' from the other side, and which on Goodman's type-token account must itself be a letter necessarily distinct from the normal English letters ' $b$ ' and ' $d$ '.

Finally, here is an equally damaging criticism. It is part of the concept of a word in any language that it is a sequence of well-defined letters or characters ${ }^{16}$ in the relevant language, and such that, for any letter in the sequence defining a word, one can unequivocally give the ordinal position of the given letter in the sequence (as the first, or second, and so on, letter). However, this cannot be done either for Goodman's deviant new ' $\mathrm{d} / \mathrm{b}$ ' letter, or for the normal letter ' $\mathrm{n}$ '.

14 Goodman, ibid., 139, fn. 7.

15 The relevant 'readings' are of course syntactic rather than semantic readings - in the case of my example, readings of the inscription as the words 'din' and 'nib'.

16 This would include characters in a pictographic language such as Chinese, many of whose characters are words in their own right - but that is true of the indefinite article ' $a$ ' in English as well. 
Thus there could not be any such word as that which presumably Goodman would have had to hypothesize to exist in the current 'din/nib' case, and hence I conclude that any type-token view of notation, that invokes a syntactic disjointness condition for inscriptions, must fail because of the possibility of such examples.

\section{A Reconstructed Notational Theory}

In conclusion, here are some brief suggestions as to how a logically adequate notational theory might be reconstructed, in light of the above failures. ${ }^{17}$

To begin, it is still important to retain a (reconstructed) form of Goodman's syntactic finite differentiation principle, ${ }^{18}$ so that, for instance, a letter ' $b$ ' as seen on one side of a transparent sign must be visually distinguishable from a letter ' $\mathrm{d}$ ' as seen on the other side of the same physical inscription.

Next, in place of the more standard type-token (or kind-instance, or universal-particular) analysis of notation, I would propose a representational account: different visual or perceptual aspects of a given physical inscription each represent a different letter, word, or other notational item.

Such an account would differ from type-theoretic accounts in at least two ways. First, the relevant perceptual aspects would not be purely physical entities (such as physical inscriptions or time-slices), in that arguably perception of an inscription as representing, from some spatio-temporal point of view, a notational item would involve an irreducibly cultural - relative to some linguistic community that uses the notation in question - perceptual interpretation of the inscription. Or, otherwise put, the relevant representational properties of an inscription, in virtue of which it represents a notational item, are not purely intrinsic, physical properties, but instead they are extrinsic, culturally determined properties.

Some indirect support for this claim is provided by Hugly and Sayward (1981, 186), who argue that the relation "being a token of" cannot be a two-place relation between a token and a type, but instead must be a four-place relation between a token, an expression, a language and a 'tokening system'. (See also the useful discussion of that paper in Simons 1982, who is skeptical both about the ontological status of types and the utility of the type-token distinction as applied to language - views which accord well with the present, alternative representational approach).

And second, arguably inscriptions bear a completely conventional representational relation to the relevant notational items, which is incompatible with any broadly realist view of type-token, kind-instance or universal-particular relations for notational items. ${ }^{19}$ For example, any text, such as the word 'nib', can readily be encrypted using other letters such as 'xyz'; but that inscription ' $x y z$ ', in the relevant interpretive context, represents exactly the same letters 'n', 'i' and 'b', and the same word 'nib' as does the inscription 'nib' itself. To be sure, we are more familiar with the usual inscriptional representations of those letters and that word, but such differences remain merely conventional.

17 A more comprehensive account is given in my paper "The Non-Typehood of Linguistic Notation", submitted for publication.

18 See fn. 5 .

19 To be sure, Goodman himself is a nominalist, but his theory is nevertheless a (nominalistic version of) a fundamentally realist rather than conventionalist analysis of notational items and their inscriptions. 
In support of this view, arguably notational items themselves can be identified purely in terms of their functional properties and relations in textual sequences, such as the fact that the letter ' $b$ ' is the third letter in the word 'nib', which word has a semantics, a well-defined extensional pattern of occurrence in historical texts, and so on, all of which properties and relations hold quite independently of our usual inscriptional representations of those items. Shakespeare's play Macbeth remains the same text (the same sequence of letters and words) even when conventionally represented in ASCII code $(A=65$, and so on $)$ and then stored in a computer, using electronic states of memory chips as the relevant inscriptions, with the aid of a further, binary representation of the relevant numbers.

If this conventionalist, representational view of notation is correct, there would be some significant implications not just for the philosophy of language, but also more generally. For example, purely formalist approaches to the philosophy of logic and mathematics would immediately look more suspect, in that even their notational preliminaries would be subject to a layer of representational interpretation. And the usual contrasts in the philosophy of mind between semantic versus (supposedly) 'purely syntactic' items and functional relations would also need to be comprehensively re-thought and reexpressed.

But in any case, some such alternative theory of notation must be found, given the failure of Goodman's fairly typical and representative type-token theory.

On a broader horizon, the present discussion is relevant also to significant metaphysical issues, such as Locke's thesis that no two things of the same sort can be in the same place at the same time (see, e.g., Fine 2000), which thesis is closely related to Goodman's type-token notational thesis that no two things of the same type - i.e., different notational characters - could be thus co-located.

Now my strategy has been, not to attack Goodman's thesis itself as a correct principle about types and tokens in general, but instead to show that the principle is inapplicable to some actual cases of notation, hence requiring an alternative representational account of inscription-notation relations. This raises the interesting possibility that a parallel strategy could be applied to some of the standard issues of material constitution, coincidence under a sortal, and so on, a strategy that would accept Locke's broader thesis as valid, and then explain away apparent counter-examples in representational terms.

For example, Kit Fine in the above-mentioned paper has argued that a single sheet of paper, on each side of which a different letter has been written (in this case, a complete communication rather than a single character), provides a counter-example to Locke's thesis, in that the two distinct letters have the same spatio-temporal location. ${ }^{20}$

However, an alternative representational account of the example could be developed along very similar lines to those discussed here, according to which there is only a single 'thing' or physical object in such a case, namely the doubly marked sheet of paper, but which object is able to represent two distinct linguistic items, namely the separate sequences of sentences making up each letter. But a more comprehensive development of this approach will have to be pursued elsewhere. ${ }^{21}$

20 Fine, ibid. My own two-sided sign case was developed independently of Fine's interesting example.

21 My thanks to Philipp Keller, and to two anonymous referees, for very helpful comments on an earlier draft of this paper. 


\section{REFERENCES}

Fine, K. 2000, “A Counter-Example to Locke's Thesis", The Monist 83, pp. 357-361.

Goodman, N. 1966, The Structure of Appearance, 2nd. ed., New York: Bobbs-Merrill.

Goodman, N. 1968, Languages of Art, New York: Bobbs-Merrill.

Hartshorne, C. and Weiss, P., eds. 1931-1958, Collected Papers of Charles Sanders Peirce, Cambridge, MA: Harvard University Press.

Hugly, P. and SAYwARD, C., 1981, "Expressions and Tokens", Analysis 41, 181-187.

SimONS, P. 1982, "Token Resistance”, Analysis 42, 195-203.

Walton, K. 1971, "Languages of Art: An Emendation”, Philosophical Studies 22, pp. 82-85. 